\title{
Ensinando a Identificação de Caracteres Braille utilizando Dispositivos Móveis e um Display Braille
}

\author{
Victor Hazin da Rocha - vhr@cin.ufpe.br \\ Diogo Silva - diogo.angra@gmail.com \\ Alvaro Boa Vista Maia Bisneto - abvmb@cesar.org.br \\ Guthemberg Felipe da Silva - gfs@ cesar.org.br \\ Fernando da Fonseca de Souza - fdfd@cin.ufpe.br
}

RESUMO: O número de deficientes visuais no mundo aumentou nos ultimos anos, contudo a taxa de alfabetização dessa parte da população vem caindo consideravelmente. Sendo um dos principais fatores para esse problema, a falta de instrutores aptos a alfabetizar pessoas em Braille. Neste contexto, este artigo apresenta o Braille Reader Tutor, uma aplicação para smartphones e um display Braille open souce, capaz de ensinar a identificação de letras e caracteres Braille, sua correspondência para o alfabeto romano, sem a necessidade de um tutor humano. Diante do exposto, foi feita uma pesquisa com profissionais especialistas em ensino de Braille para a avaliação do método proposto. Por fim, o aplicativo e o display foram desenvolvidos e disponibilizados para avaliação de profissionais responsáveis por afalbetizar deficientes visuais.

Palavras-chave: Software Educacional, Acessibilidade, Display Braille, Deficiência Visual

\section{Teaching Braille Character Identification Using Mobile Devices and a Braille Display}

ABSTRACT : The number of the visually impaired in the world has increased in recent years, but the literacy rate of this part of the population has been falling considerably. And one of the main factors causing this problem is the lack of instructors able to literate people in Braille. In this context, this paper introduces Braille Reader Tutor, a smartphone application and a braille open souce display, capable of teaching Braille character identification and matching to the Roman alphabet without the need for a human tutor. It is also presents a evaluation with professional Braille teaching experts to analyse the proposed solution. Finally, the application and display were developed and made available for evaluation by professionals responsible for impairing the visually impaired.

Keywords: Educational Software, Accessibility; Refreshable Braille display; Visual impairment;

\section{Introdução}

De acordo com dados da Organização Mundial da Saúde (OMS) há cerca de 1 bilhão de pessoas com 15 anos ou mais que vivem com algum tipo de deficiência. Dentre esses, aproximadamente 253 milhões sofrem com problemas graves de visão, dos quais aproximadamente 36 milhões de pessoas são consideradas completamente cegas (WHO, 2015). A OMS ainda estima que 19 milhões de crianças (com idade inferior a 15 anos) tenham problemas de visão, dos quais cerca de 1,4 milhão têm cegueira irreversível, 
exigindo acesso a serviços de reabilitação visual para otimizar o funcionamento e reduzir a incapacidade (WHO, 2015).

Alguns estudos afirmam que esse número pode triplicar devido ao crescimento populacional e ao envelhecimento da população. Dessa forma, até 2050, pode haver mais de 115 milhões de pessoas cegas, em comparação com a projeção de 38,5 milhões em 2020 (BOURNE et al., 2017; WHO, 2015).

Enquanto aumenta o número de deficientes visuais no mundo, a taxa de alfabetização dessa parte da população vem caindo consideravelmente nos últimos anos. Nos Estados Unidos, por exemplo, o percentual de indivíduos cegos capazes de ler em Braille é por volta de 12\% enquanto na década de 1960 era de 50\% (PUTNAM; TIGER; FICHTNER, 2015; SCHEITHAUER; TIGER, 2012; TOUSSAINT et al., 2017). O cenário é ainda pior quando se considera que mais de $90 \%$ dos deficientes visuais vivem em países em desenvolvimento, como o Brasil, onde a taxa de alfabetização desses deficientes visuais é menor que 10\% (AWANG DAMIT et al., 2014; WAGH et al., 2016).

Entre os motivos para esse número tão preocupante estão: o uso cada vez maior de leitores de tela já integrados nos smartphones, a falta de professores proficientes no ensino de Braille, bem como a falta de treinamento para professores que possuem em suas turmas crianças cegas. Além do descaso de muitos educadores, os quais não acham que a instrução em Braille é necessária (AWANG DAMIT et al., 2014; NATIONAL FEDERATION OF THE BLIND, 2015; PUTNAM; TIGER; FICHTNER, 2015; WAGH et al., 2016).

Uma vez que a leitura e a escrita ocupam um papel central na sociedade, sendo habilidade indispensável para se conseguir um emprego, é indispensável para a autonomia de um deficiente visual que o mesmo seja alfabetizado (AWANG DAMIT et al., 2014; TOUSSAINT et al., 2017). A alfabetização em Braille para pessoas com deficiência visual tem o mesmo papel que os métodos educacionais tradicionais têm para pessoas sem problemas de visão. A educação inclusiva para deficientes visuais tem sido uma preocupação mundial. $\mathrm{O}$ rápido desenvolvimento tecnológico vem criando desafios adicionais para essa parcela da população, especialmente, para aqueles que não possuem conhecimentos básicos como ler e escrever.

No processo de aprendizado de Braille, a primeira habilidade que precisa ser desenvolvida é a identificação dos caracteres lidos e a sua correspondência para o alfabeto romano (PUTNAM; TIGER; FICHTNER, 2015). Os caracteres Braille podem ser mapeados um-a-um para os números e letras que formam o alfabeto romano. Além das 26 letras, dos números e sinais de pontuação, o método Braille inclui 187 contrações para palavras comuns e combinações de letras.

Esse artigo apresenta o Braille Reader Tutor, uma aplicação para smartphones em conjunto com um display Braille open source (criado específicamente para esse fim). Tal dispositivo é capaz de ensinar a identificação dos caracteres Braille e sua correspondência para o alfabeto romano sem a necessidade de um tutor humano.

\section{Fundamentação Teórica}

Nessa seção, serão apresentadas informações importantes sobre o sistema Braille, o funcionamento de displays braille dinâmicos além do processo de alfabetização em Braille. Por fim serão apresentados alguns trabalhos relacionados relevantes. 


\subsection{O Sistema Braille}

O sistema de escrita em relevo, conhecido pelo nome de "Braille", é um sistema de leitura para cegos criado por Louis Braille em 1824, baseado em 63 símbolos em relevo resultantes da combinação de até seis pontos dispostos em duas colunas de três pontos cada. O espaço por ele ocupado, ou por qualquer outro sinal, denomina-se cela Braille ou célula Braille e, quando vazio, é também considerado por alguns especialistas como um sinal, passando assim a ser composto com 64 sinais (BRASIL, 2006).

Para facilmente se identificarem e estabelecer exatamente a sua posição relativa, os pontos são numerados de cima para baixo e da esquerda para a direita. Os três pontos que formam a coluna ou fila vertical esquerda têm os números $1,2,3$; enquanto os que compõem a coluna ou fila vertical direita possuem os números $4,5,6$.

Figura 1 - Alfabeto em Braille

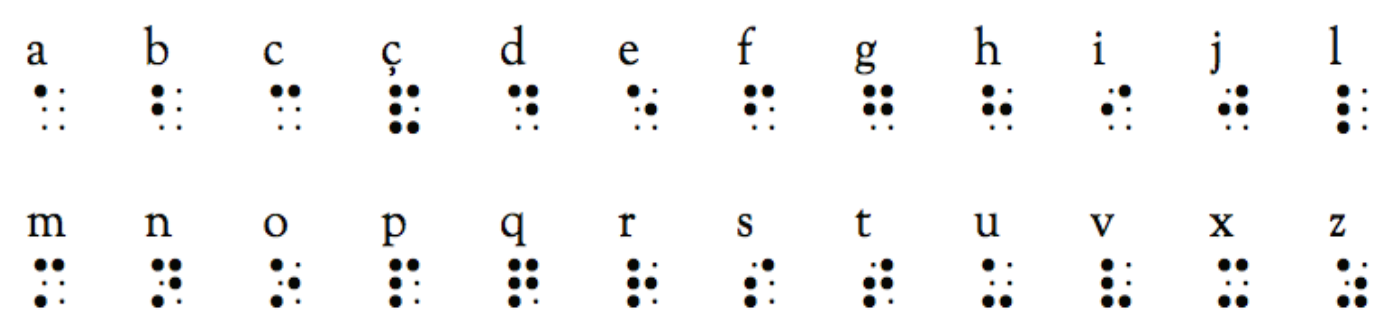

Fonte: BRASIL (2006)

O Sistema Braille é o processo de escrita em relevo mais adotado em todo o mundo e se aplica não só à representação dos símbolos literais, mas também à dos matemáticos, químicos, fonéticos, informáticos, musicais, entre outros. Na sua aplicação à Língua Portuguesa, quase todos os sinais conservam o seu significado original, a Figura 1 mostra como o alfabeto em português é exibido no sistema Braille. Apenas algumas vogais acentuadas e outros símbolos se representam por sinais que lhe são exclusivos (BRASIL, 2006).

\subsection{Displays Braille}

Displays Braille são dispositivo de saída tátil que exibem dinamicamente em Braille a informação de uma tela ligada a uma porta de saída de um computador. Em sua maioria são dispositivos eletromecânicos que apresentam os caracteres Braille pela movimentação de pinos com pontas arredondadas através de furos em um superfície plana. As forma mais utilzadas para movimentar os pinos são: a utilização de cristais piezoelétricos e mecanismos eletromagnéticos (REIS, 2013).

Nos displays eletromagnéticos cada pino é rodeado por um invólucro cilíndrico que contém uma bobina. O pino é ligado a uma mola e também a uma haste de ferro a qual passa através do invólucro. $\mathrm{O}$ conjunto funciona como um solenóide em miniatura. Quando passa uma corrente pela bobina, o campo magnético gerado atrai a haste de ferro e o pino baixa. Quando não há corrente passando pela bobina, a mola mantem o pino levantado (REIS, 2013).

Já nos displays piezoeléctricos, cada pino é montado sobre uma peça de metal que por sua vez está ligada a um cristal piezoelétrico. Se uma tensão suficientemente grande é aplicada sobre o cristal, este torna-se ligeiramente mais curto. Isto faz com que a peça de metal se curve para cima, levantando o pino. Por outro lado, na ausência de tensão, o cristal volta a sua dimensão normal desfazendo a curvatura da peça de metal e, V. $17 \mathrm{~N}^{\mathrm{o}}$ 3, dezembro, 2019 RENOTE DOI: 
consequentemente, fazendo o pino baixar. As células pizoelétricas são mais utilizadas por serem comercialmente mais disponíveis, mais leves, menores e mais fácil de controlar, o que simplifica o circuito de controle (REIS, 2013).

Com o fim de comparar os diferentes tipos de displays Braille existentes, e com isso indicar o melhor caminho para o desenvolvimento de displays mais baratos, Russomanno et al (2015), executaram um experimento avaliando a eficiência da leitura de deficientes visuais nos diferentes tipos de displays.

Do mesmo modo, após a realização dos experimentos os autores demonstraram que restringir o "contato" durante a leitura do Braille leva a um desempenho pior em uma tarefa de reconhecimento de letras. Também foi descoberto que a falta de "contato deslizante" entre a ponta do dedo e a superfície de leitura do Braille resulta em mais erros e que o número de erros aumenta em função da velocidade da apresentação (RUSSOMANNO et al., 2015).

\subsection{Alfabetização em Braille}

O processo de alfabetização no sistema Braille tem como objetivo principal o desenvolvimento da leitura com os dedos e possui também a finalidade de produzir textos manualmente. Ao iniciar a aprendizagem do Sistema Braille o aluno já deverá ter passado por um período preparatório, visando o desenvolvimento da coordenação motora fina e da percepção tátil para discriminar as formas das letras, pois as distinções são muito leves (FAÇANHA et al., 2012).

O professor trabalha com objetos concretos, sejam eles pré-fabricados ou desenvolvidos pela vivência em sala de aula, mostrando a idéia da forma das letras e levando o aluno a experimentar e traçar as letras com os dedos. Alguns objetos bem interessantes são o alfabeto, ou mesmo, alfanumérico Braille feitos de borracha de Etil Vinil Acetato - EVA e/ou de Fibra de Média Densidade (Medium Density Fiberboard MDF) (FAÇANHA et al., 2012).

Os programas convencionais para o ensino de Braille são iniciados por um programa de leitura introdutório onde primeiro é ensinado como reconhecer uma única letra em Braille. Na sequência, são apresentadas palavras de dois caracteres e em seguida palavras curtas. Palavras mais longas e frases curtas são gradualmente introduzidas com abreviações e contrações. Uma vez que textos curtos são introduzidos, é incentivada a prática da leitura para que seja possível a leitura rápida e uso de duas mãos na discriminação tátil das letras (JARJOURA; KARNI, 2014). Esse método de ensino consome uma quantidade considerável de tempo no ensino de um grupo de estudantes, pois cada aluno precisa ser ensinado individualmente (GANDHI; THAKKER; JHA, 2016).

Após execução das atividades que permitem reconhecimento das células Braille, se passa a usar a reglete e o punção como instrumento de escrita. $\mathrm{O}$ reconhecimento da combinação dos pontos apresentará uma letra, que por sua vez combinadas formará palavras. O Braille permite estudar os quadros em relevo e ler eficientemente os livros técnicos. É, ainda, o único meio de leitura disponível para os surdos-cegos. No mesmo sentido, a perfeição na escrita está relacionada com a leitura Braille que cada um faz, pois é por meio dela que o deficiente visual entra em contato com a estrutura dos textos, a ortografia das palavras e a pontuação (FAÇANHA et al., 2012) (JARJOURA; KARNI, 2014).

A qualidade do ensino do Braille é decisiva para uma leitura deste sistema e para a aquisição de hábitos de leitura. Se os alunos forem motivados à prática normal e constante do seu método de leitura e escrita, a leitura poderá ser rápida e tornar-se também mais 
agradável e instrutiva (FAÇANHA et al., 2012).

\subsection{Trabalhos Relacionados}

No artigo Teaching Identity Matching of Braille Characters To Beginning Braille Readers, os autores apresentam e avaliam um processo para ensinar incialmente a discernir as letras compostas de muitos pontos, das com poucos pontos, e gradativamente a diferença entre o número de pontos vai sendo reduzida até que a pessoa possa discernir e identificar todas as letras (TOUSSAINT et al., 2017)..

$\mathrm{O}$ artigo A Computer-Based Program to Teach Braille Reading to Sighted Individuals apresenta uma avaliação do programa de treinamento em identificação de letras em braille para indivíduos com visão, usando procedimentos instrucionais semelhantes aos de Toussaint e Tiger (2010). A grande inovação dos autores é que um programa de computador será usado para conduzir o treinamento, em vez de um instrutor humano (SCHEITHAUER; TIGER, 2012).

Em Braille Teaching Electronic Prototype, os autores apresentam um protótipo de um dispositivo eletrônico com o objetivo de ensinar a o reconhecimento de letras em Braille sem a necessidade de um tutor. O protótipo interage com o usuário através de sons e do tato. O sistema diz ao usuário qual letra está sendo exibida e em seguida apresentada no dispositivo para que o usuário possa efetuar a leitura da letra (PAUTA; VÉLEZ; SERPAANDRADE, 2016).. O problema nesse trabalho é a falta de uma metodologia apropriada para o ensino das letras de forma sequencial

\section{SOLUÇÃO PROPOSTA}

O BRT (Braille Reader Tutor) é uma solução que permite ao usuário aprender a ler em Braille um dispositivo móvel. A solução consiste em um aplicativo Android e um display em Braille open source de baixo custo. Durante o projeto três objetivos principais estiveram mente: acessibilidade, disponibilidade e baixo custo. O display do BRT foi projetado para que possa ser construído por qualquer pessoa, sem a necessidade de conhecimento especializado de microcontroladores, daí o uso do Arduíno. O aplicativo em execução no smartphone atua como uma interface para o usuário interagir com o display Braille. O aplicativo foi projetado para atender aos critérios de design universal, contendo gestos específicos independentes da visão, como deslizar para os lados, instruções de voz, vibrações e reconhecimento de fala.

\subsection{Display}

O display Braille desenvolvido neste trabalho foi baseado no funcionamento de um contador numérico manual. Cada célula Braille foi dividida em dois blocos: os três pontos que formam a coluna ou fila vertical esquerda têm os números $1,2,3$; enquanto os que compõem a coluna ou fila vertical direita possuem os números $4,5,6$. Para cada um dos blocos foram mapeadas todas as possíveis combinações dos 3 pontos (tanto em alto relevo quanto sem), o que deu um total de 8 possibilidades para cada bloco (as combinações possíveis podem ser vistas na Figura 2). 
Figura 2 - Combinações possíveis dos pontos Braille

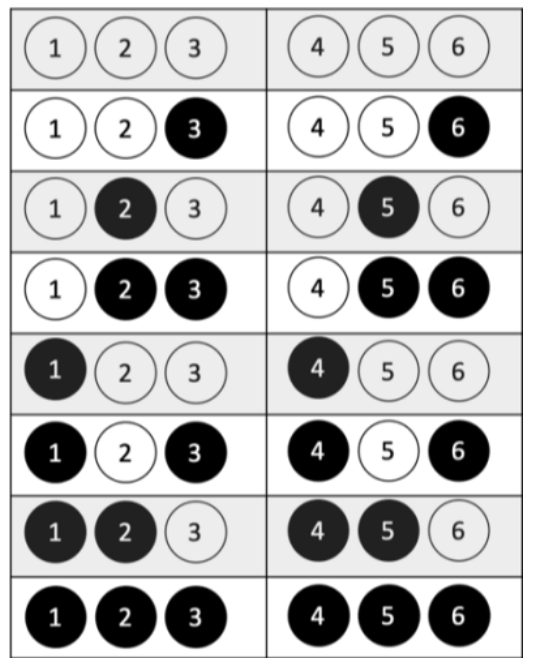

Fonte: Elaborado pelo autor (2019)

Como não existe diferença entre os 3 pontos do primeiro bloco e os do segundo bloco, a mesma peça que pode representar os pontos 1,2 e 3 pode ser utilizada para representar o segundo bloco (com os pontos 4, 5 e 6). Com isso, foi criado um modelo 3D similar a um "octógono regular estrudado" (Figura 3) no qual cada lado/face representa uma das 8 combinações possíveis. Combinando duas peças dessas é possível reproduzir todos os 64 símbolos do sistema Braille tradicional. Desse modo. duas peças iguais a da Figura 3 foram impressas utilizando uma impressora 3D Ultimaker 2.

Figura 3 - Modelo 3D

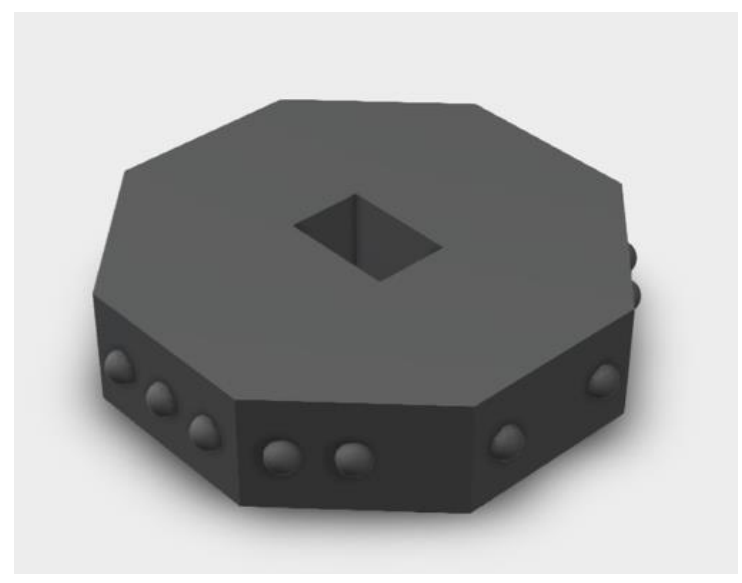

Fonte: Elaborado pelo autor (2019)

Depois disso cada "octógono" foi preso a um motor de passo (modelo 28BYJ-48) como pode ser visto na Figura 4. Cada um dos motores foi ligado a um driver ULN2003, o qual por sua vez é controlado por um Arduíno nano ATmega328.

Também foi ligado ao Arduíno um bluetooth HC-06 para que as "letras", as quais devem ser exibidas no display sejam enviadas para ele por essa via. Elimina-se assim a necessidade de uso de fios para ligar o display a um computador ou smartphone. Os motores foram presos a um suporte e colocados dentro de uma caixa para impedir o contato direto dos usuários com os componentes eletrônicos. Tanto o suporte quanto a caixa foram modelados sob medida e impressos utilizando a Ultimaker 2 e podem ser vistos na Figura 4. 


\section{Figura 4 - Display Braille Open Source}

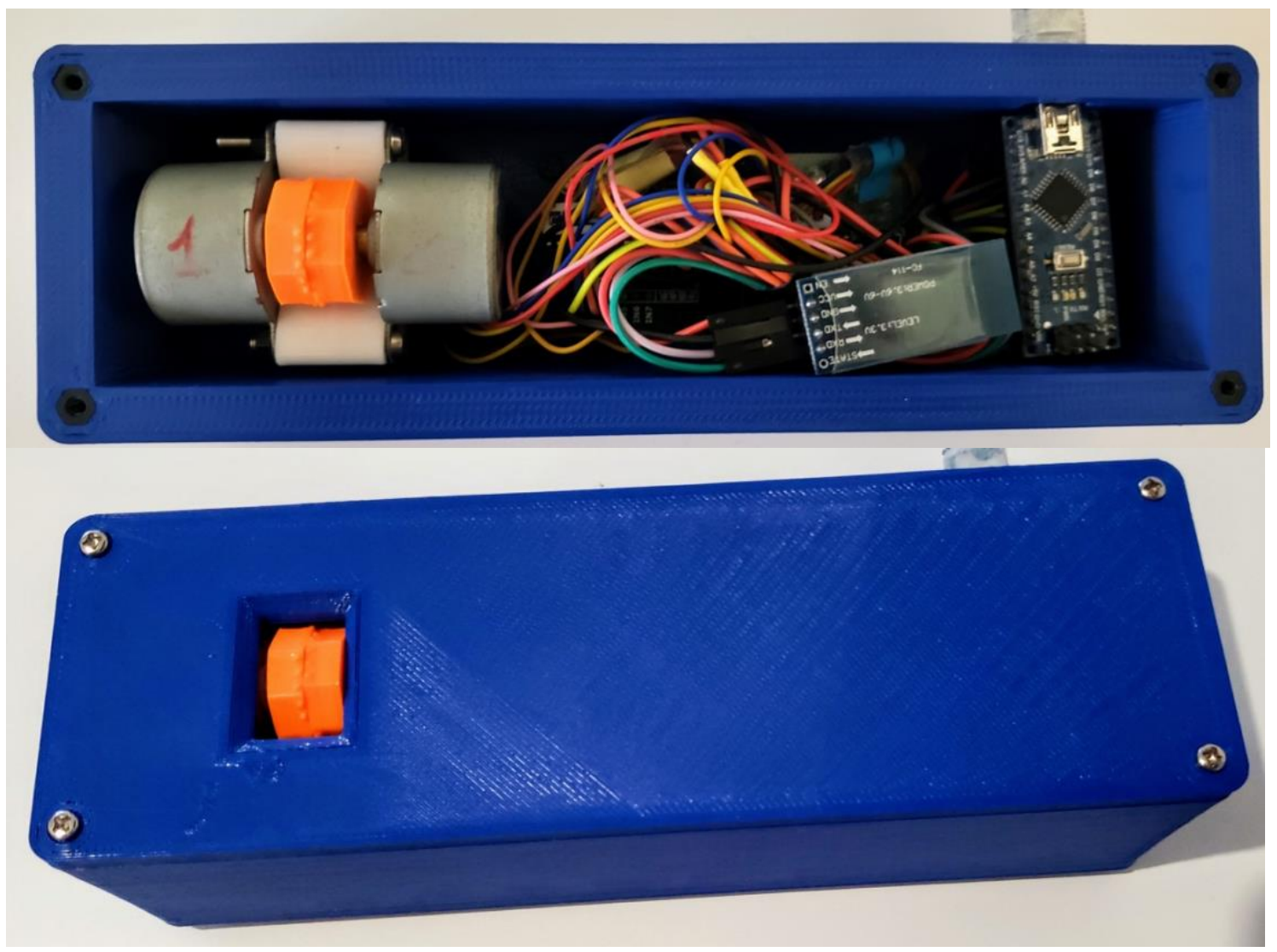

Fonte: Elaborado pelo autor (2019)

\subsection{Aplicação}

A aplicação é para a plataforma Android, uma vez que o custo de aparelhos que suportam essa plataforma é mais baixo que os que suportam o iOS. A aplicação consiste em duas partes, uma com o objetivo de apresentar e ensinar ao usuário a representação em Braille de cada letra do alfabeto, enquanto a outra parte tem o intuito de avaliar se o usuário é capaz de reconhecer os caracteres em Braille. Cada parte é dividida em 6 módulos, inspirados pelos trabalhos de Toussaint et al. (2017). Na Figura 5 é possível ver as telas da aplicação.

A parte de ensino é chamada Alfabeto no aplicativo (terceira imagem da Figura 5) e apresentará cada letra do módulo em sua representação em Braille. A tela contém 3 botões coloridos grandes para facilitar o uso por pessoas com deficiência visual e com baixa visão. O primeiro botão retorna à tela inicial do aplicativo, enquanto o segundo botão possui três funções diferentes: navegar pela lista de letras desse módulo (usando deslizar para a esquerda / direita); enviar a letra selecionada para a tela (com um toque duplo no botão) e para ouvir a letra selecionada atual (toque único). Por fim, o terceiro botão fala ao usuário qual letra que está sendo exibida no visor em Braille.

A parte de avaliação é chamada de "Qual é a letra"? (quarta imagem da Figura 5) e é semelhante à tela de ensino, mantendo a mesma estrutura de três botões grandes. $\mathrm{O}$ objetivo de manter a mesma estrutura, é facilitar a usabilidade do aplicativo, criando telas e comandos semelhantes, para que os usuários possam memorizar melhor as maneiras de interagir com o aplicativo. O primeiro e o terceiro botão, funcionam da mesma forma que na outra tela. O segundo botão possui três funções diferentes: deslizando para 
direita/esquerda o usuário pode navegar pela lista de letras desse módulo; com um toque duplo a letra selecionada é enviada para a tela e com um toque único a aplicação vai solicitar que o usuário fale a letra que ele leu display Braille.

Figura 5 - Telas da Aplicação (da esquerda pra direita: Tela Inicial, Tela de seleção de modulo, Tela de Ensino, Tela de Avaliação)

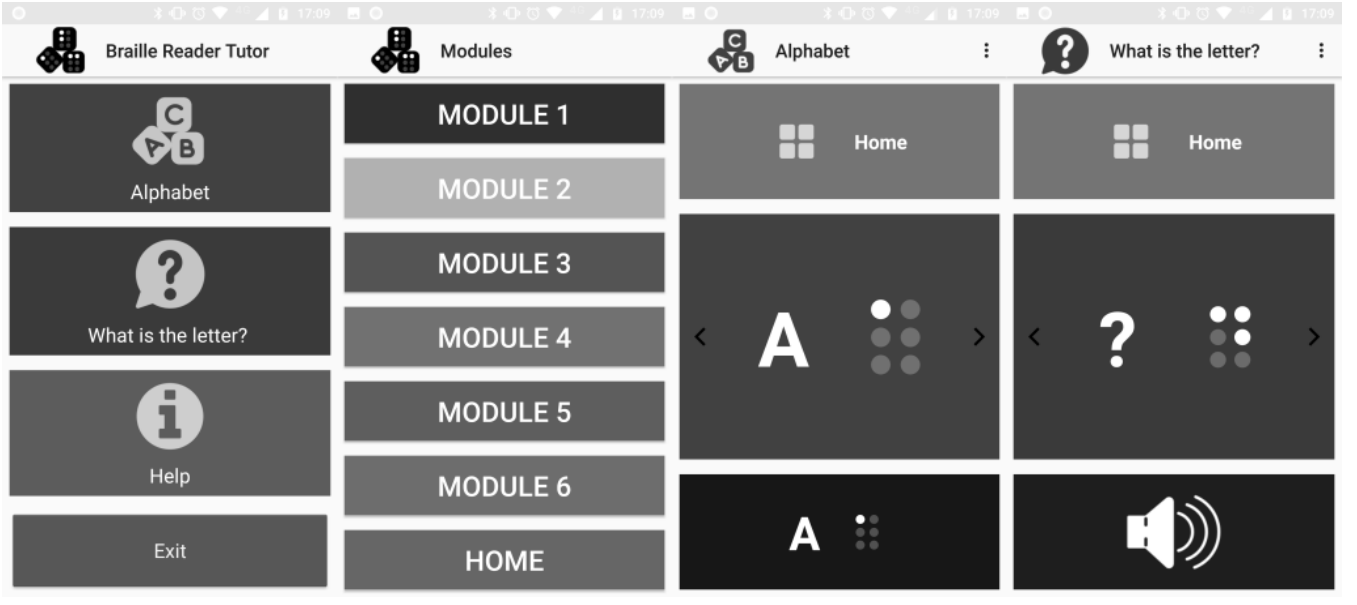

Fonte: Elaborada pelo autor (2019)

Os módulos são divididos da seguinte forma: $O$ primeiro contém a única letra que é representada com apenas um ponto (A); o o segundo abrange as cinco letras representadas com dois pontos (B, C, E, I e K); o terceiro inclui as nove letras representadas com três pontos (D, F, H, J, L, M, O, S e U); o quarto é composto pelas nove letras representadas com quatro pontos ( $\mathrm{G}, \mathrm{N}, \mathrm{P}, \mathrm{R}, \mathrm{T}, \mathrm{V}, \mathrm{W}, \mathrm{X}$ e $\mathrm{Z})$; o quinto engloba as duas letras representadas com cinco pontos ( $\mathrm{Q}$ e $\mathrm{Y})$; e o módulo 6 contém todas as letras. Esse modelo de divisão por módulos foi inicialmente proposto por Toussaint et al. (2017).

O usuário deve primeiro concluir o módulo de ensino e, em seguida, o módulo de teste correspondente, o acesso aos módulos a seguinte será liberado somente após a conclusão dos módulos anteriores.

\section{VALIDAÇÃ̃o E DISCUSSÃO}

Para validar a proposta, foram feitos testes iniciais com dois voluntários no Instituto de Cegos Antônio Pessoa de Queiroz, em Recife, Brasil. Ambos os voluntários trabalham e estão familiarizados com o processo de ensino de Braille. Durante os testes, os voluntários foram incentivados a dar feedback sobre o uso do protótipo e falar sobre a legibilidade da tela. Em relação ao protótipo, os usuários comentaram que o acharam interessante e empolgados com o custo da solução, uma vez que os displays comerciais em Braille custam mais de 2000 dólares.

Foram detectadas algumas divergências entre a solução proposta com apenas uma célula e a leitura tradicional em Braille. Por exemplo: as distâncias dos pontos da célula devem ser ajustadas para reduzir a distância entre as duas colunas, pois causou a impressão de que cada coluna era uma letra separada da outra. Por outro lado, algumas das vantagens da solução Braille de uma célula são: a leitura estática reduz a fadiga, também o preço de um equipamento celular é mais baixo, a simplicidade, a falta de problemas de quebra de linha porque a mão permanece estática sobre o dispositivo, além da manutenção ser mais simples . 
A solução proposta, ainda encontra-se em testes tanto com deficientes visuais quanto com professores, uma vez que a identificação de caracteres Braille é uma das habilidades fundamentais que para professores que possuem deficientes visuais nas suas turmas precisam possuir (PUTNAM; TIGER; FICHTNER, 2015; TOUSSAINT et al., 2017). O que mostra como é fundamental o desenvolvimento de soluções para ensinar aos professores as habilidades necessárias, e assim fornecer instruções em Braille, área pouco abordada por pesquisas tanto na área de acessibilidade quanto de educação (PUTNAM; TIGER; FICHTNER, 2015).

\section{CONSIDERAÇÕES FINAIS}

O problema principal que norteou esta pesquisa foi o fato de que apenas uma pequena parte da população de deficientes visuais é proficiente na leitura em Braille (AWANG DAMIT et al., 2014; WAGH et al., 2016). A leitura e a escrita ocupam um papel central na sociedade, sendo habilidade indispensável para se conseguir um emprego, dessa forma é imprescindível para a autonomia de um deficiente visual que o mesmo seja alfabetizado. Um dos principais fatores para esse déficit é a falta de instrutores aptos a alfabetizar pessoas em Braille (AWANG DAMIT et al., 2014; NATIONAL FEDERATION OF THE BLIND, 2015; PUTNAM; TIGER; FICHTNER, 2015; WAGH et al., 2016).

Com base nessa constatação, esse trabalho apresenta o BRT, uma aplicação para smartphones em conjunto com um display Braille open source (criado específicamente para esse fim), capaz de ensinar a identificação dos caracteres Braille e a sua correspondência para o alfabeto romano sem a necessidade de um tutor humano. Outra contribuição desse trabalho é o display Braille, open source, desenvolvido utilizando Arduíno e impressão 3D. Dessa forma, o display pode ser utilizado para outros fins.

Como trabalho futuro o está previsto o desenvolvimento das melhorias sugeridas durante a avaliação, assim como novas rodadas de testes da solução proposta com mais voluntários.

\section{REFERÊNCIAS BIBLIOGRÁFICAS}

AWANG DAMIT, Dayang Suhaida et al. Dual braille code translator: Basic education tool for visually impaired children. In: 2014 INTERNATIONAL CONFERENCE ON COMPUTER, COMMUNICATIONS, AND CONTROL TECHNOLOGY (I4CT) 2014, Langkawi. Anais... Langkawi: IEEE, 2014. Disponível em:

$<$ http://ieeexplore.ieee.org/lpdocs/epic03/wrapper.htm?arnumber=6914213>

BOURNE, Rupert R. A. et al. Magnitude, temporal trends, and projections of the global prevalence of blindness and distance and near vision impairment: a systematic review and metaanalysis. The Lancet Global Health, [s. 1.], v. 5, n. 9, p. e888-e897, 2017. Disponível em: $<$ http://linkinghub.elsevier.com/retrieve/pii/S2214109X17302930>

BRASIL. Ministério da Educação. Secretaria de Educação Especial. Grafia a Braille para a Língua Portuguesa.Brasília, 2006.

FAÇANHA, Agebson Rocha et al. Auxiliando o Processo de Ensino-Aprendizagem do Braille Através de Dispositivos Touch Screen. Informática na educação: teoria \& prática, [s. 1.], v. 15, n. 2, p. 153-169, 2012. Disponível em:

$<$ https://seer.ufrgs.br/InfEducTeoriaPratica/article/view/23197>

GANDHI, Shreya; THAKKER, Bhaskar; JHA, Shreelal. Braille cell actuator based teaching 
system for visually impaired students. In: 2016 IEEE INTERNATIONAL CONFERENCE ON RECENT TRENDS IN ELECTRONICS, INFORMATION \& COMMUNICATION TECHNOLOGY (RTEICT) 2016, Bangalore. Anais... Bangalore: IEEE, 2016. Disponível em: $<$ http://ieeexplore.ieee.org/document/7808057/>

JARJOURA, Waleed; KARNI, Avi. Braille Reading in Blind and Sighted Individuals: Educational Considerations and Experimental Evidence. In: [s.l: s.n.]. p. 395-408. NATIONAL FEDERATION OF THE BLIND. How many children in America are not taught to read? 2015. Disponível em: $<\mathrm{https}: / / \mathrm{hfb}$.org/braille-initiative $>$. Acesso em: 16 abr. 2018.

PAUTA, Jorge Andrés Acuay; VÉLEZ, Eduardo Pinos; SERPA-ANDRADE, Luis. Braille teaching electronic prototype. In: 2016 IEEE INTERNATIONAL AUTUMN MEETING ON POWER, ELECTRONICS AND COMPUTING (ROPEC) 2016, Anais... [s.l: s.n.]

PUTNAM, Brittany C.; TIGER, Jeffrey H.; FICHTNER, Caitlin. Teaching braille letters, numerals, punctuation, and contractions to sighted individuals. Journal of Applied Behavior Analysis, [s. 1.], v. 2, n. 2, p. 466-471, 2015.

REIS, Sérgio Valle Dos. Painel Braille Interativo. 2013. Unicamp, [s. 1.], 2013. Disponível em: <http://bdtd.ibict.br/vufind/Record/CAMP_0662d1c66acb33f770ab1344ef02e676> RUSSOMANNO, Alexander et al. Refreshing Refreshable Braille Displays. IEEE Transactions on Haptics, [s. 1.], v. 8, n. 3, p. 287-297, 2015. Disponível em: $<\mathrm{http}$ //ieeexplore.ieee.org/document/7086320/>

SCHEITHAUER, Mindy C.; TIGER, Jeffrey H. A COMPUTER-BASED PROGRAM TO TEACH BRAILLE READING TO SIGHTED INDIVIDUALS. Journal of Applied Behavior Analysis, [s. 1.], v. 2, n. 2, p. 315-327, 2012.

TOUSSAINT, Karen A. et al. Teaching identity matching of braille characters to beginning braille readers. Journal of Applied Behavior Analysis, [s. 1.], v. 50, n. 2, p. 278-289, 2017.

TOUSSAINT, Karen A.; TIGER, Jeffrey H. Teaching Early Braille Literacy Skills Within a Stimulus Equivalence Paradigm To Children With Degenerative Visual Impairments. Journal of Applied Behavior Analysis, [s. 1.], v. 43, n. 2, p. 181-194, 2010. Disponível em: $<$ http://www.ncbi.nlm.nih.gov/pmc/articles/PMC2884344/>

WAGH, Parag et al. E-Braille-a self-learning Braille device. In: 2016 TWENTY SECOND NATIONAL CONFERENCE ON COMMUNICATION (NCC) 2016, Anais... : IEEE, 2016. Disponível em: $<$ http://ieeexplore.ieee.org/document/7561162/>

WHO. WHO global disability action plan 2014-2021, 2015. Disponível em: $<$ https://www.who.int/disabilities/actionplan/en/> 